\title{
BCLC Stage
}

National Cancer Institute

\section{Source}

National Cancer Institute. BCLC Stage. NCI Thesaurus. Code C115134.

A stage for hepatocellular carcinoma defined according to the Barcelona Clinic Liver Cancer (BCLC) criteria. 